\title{
Footnote S9
}

Reliable methods are a set of instructions that are (i) consciously developed and documented, (ii) owned by someone, (iii) always followed by everyone performing the work and (iv) the basis for improvement. 